\title{
Neurologists' Assessment of Mental Comorbidity in Patients With Vertigo and Dizziness in Routine Clinical Care-Comparison With a Structured Clinical Interview
}

\section{OPEN ACCESS}

Edited by:

Jeffrey P. Staab,

Mayo Clinic, United States

Reviewed by:

Jorge Kattah,

University of Illinois College of

Medicine, United States

Fumiyuki Goto,

Tokyo Medical Center (NHO), Japan

${ }^{*}$ Correspondence: Karina Limburg

karina.limburg@psy.Imu.de

Specialty section:

This article was submitted to Neuro-Otology,

a section of the journal

Frontiers in Neurology

Received: 07 September 2018

Accepted: 25 October 2018

Published: 13 November 2018

Citation:

Limburg K, Dinkel A,

Schmid-Mühlbauer G, Sattel H,

Radziej K, Becker-Bense S,

Henningsen $P$, Dieterich $M$ and

Lahmann C (2018) Neurologists' Assessment of Mental Comorbidity in Patients With Vertigo and Dizziness in Routine Clinical Care-Comparison With a Structured Clinical Interview.

Front. Neurol. 9:957.

doi: 10.3389/fneur.2018.00957

\begin{abstract}
Karina Limburg ${ }^{1 *}$, Andreas Dinkel ${ }^{2}$, Gabriele Schmid-Mühlbauer ${ }^{3}$, Heribert Sattel ${ }^{2}$, Katharina Radziej ${ }^{2}$, Sandra Becker-Bense ${ }^{4}$, Peter Henningsen ${ }^{2}$, Marianne Dieterich ${ }^{4,5,6}$ and Claas Lahmann ${ }^{7}$

${ }^{1}$ LMU Munich, Department of Psychology, Munich, Germany, ${ }^{2}$ Department of Psychosomatic Medicine and Psychotherapy, Klinikum Rechts der Isar, Technical University Munich, Munich, Germany, ${ }^{3}$ Landeshauptstadt München, Munich, Germany, ${ }^{4}$ German Center for Vertigo and Balance Disorders, University Hospital, Ludwig-Maximilians-University, Munich, Germany, ${ }^{5}$ Department of Neurology, University Hospital, Ludwig-Maximilians University, Munich, Germany, ${ }^{6}$ Cluster of Systems Neurology-SyNergy, Munich, Germany, ${ }^{7}$ Department of Psychosomatic Medicine and Psychotherapy, University Medical Center Freiburg, Freiburg, Germany
\end{abstract}

Background: Mental health comorbidities are frequent in patients with vertigo and dizziness. The current study was conducted in a specialized interdisciplinary university center for vertigo and dizziness. Clinical routines consist of a structured work-up in which neuro-otological and neurological tests are performed to first detect possible organic vestibular deficits. In addition, psychiatric disorders and comorbidities are considered. The study aimed to evaluate neurologists' awareness of psychiatric next to somatic disorders within patients' first examination in terms of diagnostic congruence between neurologists' diagnoses and structured clinical assessment of mental disorders.

Methods: The study involved 392 patients. Diagnostic evaluation included (a) structured history-taking (including psychosocial anamnesis), neurological, and neuro-otological diagnostics conducted by neurologists and (b) a structured clinical interview for mental disorders (SCID-I) conducted by psychologists and final-year medical or psychology students. Cohen's Kappa was calculated to determine agreement rates regarding depression and anxiety disorders; additionally, sensitivity and specificity were evaluated.

Results: Neurologists' assessments led to at least one psychiatric diagnosis among the main diagnoses in 40 (10.2\%) patients, whereas the structured clinical interview led to at least one DSM-IV psychiatric diagnosis in 174 (44.4\%) of the patients. Agreement was low $(\kappa<0.2)$; sensitivity was low $(15 \%)$ but specificity was high $(98 \%)$.

Conclusions: Agreement between the diagnosis of neurologists and structured clinical interviews for psychiatric disorders is low. Since psychiatric disorders are frequent in vertigo and dizziness and tend to take a chronic course, improving early recognition and implementing appropriate care concepts is vital.

Keywords: structured clinical interviews for mental disorders, vertigo, dizziness, psychiatric disorders, diagnostic agreement 


\section{INTRODUCTION}

Vertigo and dizziness are frequently presented symptoms that are comorbid with mental disorders in about $30-50 \%$ of complex and often chronic vertigo and dizziness syndromes $(1,2)$. The most common comorbidities are depression and anxiety (3). Additionally, functional vertigo and dizziness symptoms (i.e., symptoms without any underlying structural dysfunction) are very common and often occur due to a psychiatric condition $(4,5)$. Initially, patients with vertigo and dizziness symptoms usually consult their general practitioner or a specialist from a somatic discipline, typically a neurologist or otolaryngologist. Since mental comorbidities often tend to take a chronic course if not recognized early $(6,7)$, detecting possible mental disorders early in the diagnostic process would be beneficial in order to initiate the appropriate treatment or prevention methods. However, even though mental comorbidities are frequent in many neurological disorders and are thought to complicate treatment (8), few investigations toward neurologists' awareness of psychiatric conditions in clinical routine exist. One study examining this issue found low sensitivity in neurologists' detection of depression in patients with Parkinson's symptoms (9). Findings from respective studies in other medical fields, e.g., in oncology (10), primary and secondary care regarding alcohol problems (11), and other somatic hospital departments (12) agree that the recognition and correct identification of psychiatric conditions or psychological distress is low across the disciplines. Therefore, collaborative care approaches that aim at integrating psychosomatic or psychiatric assessments and care into somatic care are becoming increasingly popular since they could assist in recognition and treatment of mental health conditions $(13,14)$.

The aim of the current study was to investigate neurologists' recognition of psychiatric diagnoses in patients with vertigo and dizziness symptoms during clinical routine, i.e., a first examination at a specialized tertiary care center. To do so, we aimed to evaluate the prevalence rates estimated by the primary diagnoses of neurologists and compare them with those detected by the current gold standard, the structured clinical interview for mental disorders after DSM-IV, SCID-I (15). In this regard, we aimed at specifically evaluating the concordance of anxiety and depressive disorder diagnoses since these are most common $(3,16)$.

\section{METHODS}

\section{Study Design, Setting, and Sample}

This secondary cross-sectional analysis of a longitudinal study was conducted between May 2010 and June 2012 and involved 687 patients who gave their informed consent (from a total of 860 eligible patients). Patients were recruited through routine care appointments at the interdisciplinary German Center for Vertigo and Balance Disorders, University Hospital Munich, Germany. Due to organizational reasons (e.g., living outside of Munich or nausea and vomiting after caloric testing), not all patients finished the time consuming psychological assessment following the neurological and neuro-otological procedures. Therefore, we were able to include and analyse complete data from 392 patients
(44.1\% male, $54.8 \pm 16.0$ years of age). The majority of these patients, i.e., almost $80 \%$ presented with chronic complaints that had persisted for at least 6 months before initial presentation at the center (17).

Patients were included if they were at least 18 years of age and were fluent with the German language. Furthermore, assessment via SCID-I and completed relevant questionnaires needed to be available. Exclusion criteria were any of the following diagnoses: neurodegenerative disorder (e.g., dementia), schizoaffective disorder, psychotic disorder, substance abuse, or severe suicidal tendencies. All participants were informed about the aims of the study and provided written informed consent. The study was approved by the Ethics Committee of the University of Munich (ref: 108-10). The design of this study has been previously described in detail by Lahmann et al. (18).

\section{Neurological Diagnostic Work-Up}

All patients underwent structured history taking and a systematic and standardized physical examination by neurologists at the German Center for Vertigo and Balance Disorders, including complete neurological, neuro-otological, neuroophthalmological, and neuro-orthoptic examination. Details on the neurological assessments are provided elsewhere (17). History taking included a psychosocial anamnesis to evaluate mental health comorbidity in an unstructured manner. However, neurologists were not explicitly instructed to screen for psychiatric disorders since the first neuro-otological examination aims at disclosing vestibular dysfunctions. Psychiatric diagnoses were assessed according to the International Classification of Diseases [ICD-10; (19)]. All diagnoses, i.e., main and secondary diagnoses, were noted in a specified section within the patients' reports; these were extracted for the current study. However, some physicians noted aspects of psychopathology (i.e., depressed mood, thought disorders, etc.) in the running text of the patient's report which were not coded in the diagnoses section.

Neurologists working at the center are residents in their second or higher year of specialized medical training. The residents are supervised by a senior neurologist specialized in neuro-otology with whom every diagnostic decision is discussed. At the time of data collection, there were seven residents and five senior neurologists working at the center. The neurologists are clinically trained in the screening of mental disorders via standardized clinical teachings and courses within the field of psychosomatic medicine, which are held by colleagues from the psychosomatic department.

\section{Psychometric Examination: Structured Clinical Interview (SCID-I)}

Psychologists and final-year medical or psychology students conducted structured clinical interviews (SCID-I) to assess patients' mental disorders and psychiatric comorbidity according to the DSM-IV classification system independently of their diagnoses given by the neurologists (15). Interviews were conducted on the same day after the neurological assessment. We used the DSM-IV classification system since data collection was conducted before the DSM-5 (20) came out. Furthermore, 
the DSM-IV is still the current clinical "gold" standard and no German translation of the SCID for DSM-5 exists yet. However, because most DSM-IV somatoform disorders have been merged into DSM-5 somatic symptom disorder (SSD) (20), we decided not to evaluate the concordance of DSM-IV somatoform disorder diagnoses from our evaluation. All interviewers underwent intensive training, which included practice interviews with patients that were not recruited for the study. Interrater reliability was evaluated via interviews with a simulated patient; Kappa was 0.94. Interviewers were required to attend a regular SCID supervision led by a senior physician (CL).

\section{Statistical Analysis}

We conducted all analyses using SPSS (version 24). We used descriptive statistics to describe the sample and to evaluate prevalence rates of diagnoses. A sensitivity analysis comparing study sample and dropout group was conducted with $t$-tests and chi-square tests. To test for differences among neurologists' psychiatric diagnoses vs. current SCID-I diagnoses, chi-square tests were conducted. Furthermore, the agreement rate between the diagnosis given by neurologists after clinical diagnostic workup and the SCID-I diagnosis was evaluated using Cohen's Kappa coefficient $(\mathrm{K})$, which was computed as follows: $\left[\left(\mathrm{p}_{\mathrm{o}}-\mathrm{p}_{\mathrm{c}}\right) /\left(1-\mathrm{p}_{\mathrm{c}}\right)\right]$, i.e., $\mathrm{p}_{\mathrm{o}}$ is the observed agreement rate and $\mathrm{p}_{\mathrm{c}}$ the agreement rate by chance. Cohen's Kappa can be classified as follows: $\mathrm{K} \leq 0.20$ no or weak agreement; $0.21 \leq \mathrm{K} \leq 0.40$ small agreement; $0.41 \leq \mathrm{K} \leq$ 0.60 moderate agreement; $0.61 \leq \mathrm{K} \leq 0.80$ high agreement; 0.81 $\leq \mathrm{K} \leq 1.00$ very high agreement (21). In addition, we investigated sensitivity and specificity of doctors' assessments as indicators of the true positive and true negative rates, respectively (22).

\section{RESULTS}

Sociodemographic and medical characteristics of the patient study sample $(n=392)$ and the group excluded from the analyses $(n=295)$ along with a sensitivity analysis regarding relevant variables are presented in Table 1. Study sample and excluded patients differed regarding age (with the study sample being significantly younger). In the neurological diagnostic workup, a total of 252 patients (64.3\%) of the study sample were diagnosed with a purely structural cause of vertigo. For the remaining 140 patients (35.7\%), the vertigo and dizziness symptoms were classified by the neurologists as having functional vertigo or a functional component. About a third of patients received more than one somatic diagnosis, thus 468 diagnoses were given overall. Of those, 144 (30.8\%) were functional vertigo and dizziness, 324 (69.2\%) included a structural dysfunction amongst the main diagnoses.

\section{Prevalence and Agreement of Psychiatric Diagnoses: Neurologists' Diagnoses Compared to SCID-I}

Table 2 presents the psychiatric diagnoses given by neurologists and those after DSM-IV and their prevalence rates. Structured clinical interview led to a total of 275 diagnoses and at least one psychiatric diagnosis after DSM-IV in $174(44.4 \%)$ of all patients;
TABLE 1 | Sociodemographic and medical characteristics of the study sample and patients excluded from the analyses.

\begin{tabular}{|c|c|c|c|c|}
\hline & \multirow[t]{2}{*}{$\begin{array}{c}\text { Study } \\
\text { sample } \\
(n=392)\end{array}$} & \multicolumn{2}{|c|}{$\begin{array}{c}\text { Excluded from } \\
\text { analyses } \\
(n=295)\end{array}$} & \multirow[t]{2}{*}{$X^{2}$ or $T$} \\
\hline & & $\begin{array}{l}n \text { (existing } \\
\text { data) }\end{array}$ & $\begin{array}{l}M(S D) \text { or } \\
n(\%)\end{array}$ & \\
\hline \multicolumn{5}{|l|}{ VARIABLE } \\
\hline Age, $M(S D)$ & $53.9(15.8)$ & 120 & $57.5(16.9)$ & $T=2.2^{*}$ \\
\hline Female gender, $n(\%)$ & $221(56.4)$ & 295 & $180(61.0)$ & $x^{2}=1.5$ \\
\hline Marital status ( $n$ \% married) & $246(62.8)$ & 119 & $74(62.2)$ & $x^{2}=8.2$ \\
\hline Education & & 120 & & $x^{2}=1.4$ \\
\hline 9th grade or less, $n(\%)$ & $161(41.1)$ & & $50(41.7)$ & \\
\hline 10th grade, $n(\%)$ & $124(31.6)$ & & $36(30.0)$ & \\
\hline $\begin{array}{l}\text { High school graduate, } \\
n(\%)\end{array}$ & $45(11.5)$ & & $14(11.7)$ & \\
\hline $\begin{array}{l}\text { University graduate, } \\
n(\%)\end{array}$ & $62(15.8)$ & & $20(16.7)$ & \\
\hline $\begin{array}{l}\text { Neurological diagnoses, } n \\
\text { (total) }\end{array}$ & 468 & 295 & 367 & \\
\hline $\begin{array}{l}\text { Functional vertigo and } \\
\text { dizziness symptoms, } n \\
(\%)\end{array}$ & $144(30.8)$ & & $119(32.4)$ & $x^{2}=0.9$ \\
\hline $\begin{array}{l}\text { Vestibular paroxysmia, } \\
n(\%)\end{array}$ & $30(6.4)$ & & $20(5.4)$ & $x^{2}=0.19$ \\
\hline $\begin{array}{l}\text { Vestibular migraine, } \\
n(\%)\end{array}$ & 75 (16.0) & & $41(11.2)$ & $x^{2}=3.3$ \\
\hline $\begin{array}{l}\text { Multisensory deficit, } \\
n(\%)\end{array}$ & $23(4.9)$ & & $27(7.4)$ & $x^{2}=2.7$ \\
\hline $\begin{array}{l}\text { Benign paroxysmal } \\
\text { positional vertigo, } n(\%)\end{array}$ & $66(14.1)$ & & 45 (12.3) & $x^{2}=0.31$ \\
\hline Central vertigo, $n(\%)$ & $24(5.1)$ & & $25(6.8)$ & $x^{2}=1.4$ \\
\hline Meniere's disease, $n(\%)$ & $57(12.2)$ & & $44(12.0)$ & $x^{2}=0.02$ \\
\hline Vestibular neuritis, $n(\%)$ & $22(4.7)$ & & $12(3.3)$ & $x^{2}=0.85$ \\
\hline $\begin{array}{l}\text { Bilateral Vestibulopathy, } \\
n(\%)\end{array}$ & $27(5.8)$ & & $34(9.3)$ & $x^{2}=4.5^{\star}$ \\
\hline \multicolumn{5}{|l|}{ PSYCHOPATHOLOGY } \\
\hline Depression (BDI-II), M (SD) & $11.4(8.2)$ & 112 & $11.9(9.1)$ & $T=0.61$ \\
\hline Anxiety (BAI), M (SD) & $13.2(9.4)$ & 115 & $14.8(10.6)$ & $T=1.53$ \\
\hline $\begin{array}{l}\text { Somatization (PHQ-15), M } \\
\text { (SD) }\end{array}$ & $9.8(4.9)$ & 116 & $9.7(5.0)$ & $T=-0.22$ \\
\hline
\end{tabular}

Multiple psychiatric and neurologic diagnoses were allowed if indicated. DSM, Diagnostic and Statistical Manual of Mental Disorders; PHQ, Patient Health Questionnaire; BDI, Beck Depression Inventory; BAI, Beck Anxiety Inventory. ${ }^{*} p<0.05$.

$66(16.8 \%)$ patients of the total sample fulfilled DSM-IV-criteria of a depressive disorder, 130 (33.2\%) patients fulfilled the criteria of at least one anxiety disorder. The neurologists' assessment led to substantially lower rates of psychiatric diagnoses: 49 diagnoses were given in total, $40(10.2 \%)$ of all patients were diagnosed with at least one psychiatric diagnosis, 15 (3.8\%) patients of the whole sample with a depressive disorder and $24(6.1 \%)$ with at least one anxiety disorder. Remaining diagnoses were substance-related and adjustment disorders. Agreement between the neurologists' diagnoses and structured clinical interviews was low throughout, with Cohen's $\kappa$ values below 0.2. A total of 10 (15.2\%) depressive and $20(15.3 \%)$ of all anxiety disorders were 
TABLE 2 | Diagnoses according to neurologists and DSM-IV.

\begin{tabular}{lcc}
\hline Diagnosis & $\begin{array}{c}\text { Neurologist } \\
\boldsymbol{n} \text { (\% of total) }\end{array}$ & $\begin{array}{c}\text { DSM-IV } \\
\boldsymbol{n} \text { (\% of total) }\end{array}$ \\
\hline Total number of psychiatric diagnoses & $49(100)$ & $275(100)$ \\
Depressive disorder & $15(30.6)$ & $66(23.9)$ \\
Anxiety disorder & $27(55.1)$ & $169(61.5)$ \\
$\quad$ Panic disorder & $15(30.6)$ & $23(8.4)$ \\
$\quad$ Panic disorder with agoraphobia & $2(4.1)$ & $25(9.1)$ \\
$\quad$ Agoraphobia without panic disorder & $2(4.1)$ & $40(14.6)$ \\
$\quad$ Social phobia & $0(0.0)$ & $14(5.1)$ \\
$\quad$ Specific phobia & $1(2.0)$ & $48(17.5)$ \\
$\quad$ OCD & $0(0.0)$ & $0(0.0)$ \\
$\quad$ PTSD & $0(0.0)$ & $9(3.3)$ \\
$\quad$ GAD & $2(4.1)$ & $10(3.5)$ \\
$\quad$ Not otherwise specified & $5(10.2)$ & $0(0.0)$ \\
Substance-related disorder & $1(2.0)$ & $31(11.3)$ \\
Eating disorder & $0(0.0)$ & $9(3.3)$ \\
Adjustment disorder & $6(12.3)$ & $0(0.0)$ \\
\hline
\end{tabular}

$O C D$, obsessive-compulsive disorder; PTSD, posttraumatic stress disorder; GAD, generalized anxiety disorder.

TABLE 3 | Agreement between doctor's rating of psychiatric disorders and DSM-IV-classification of disorders (excluding somatoform disorders).

\begin{tabular}{|c|c|c|c|c|}
\hline \multirow[t]{2}{*}{ DSM-IV } & \multicolumn{2}{|c|}{ Doctor's rating } & \multirow[t]{2}{*}{$X^{2}$} & \multirow[t]{2}{*}{ Cohen's } \\
\hline & No $(n)$ & Yes $(n)$ & & \\
\hline
\end{tabular}

\begin{tabular}{|c|c|c|c|c|}
\hline \multicolumn{5}{|c|}{ ANY PSYCHIATRIC DIAGNOSIS GIVEN } \\
\hline No $(n)$ & 210 & 8 & $22.9^{\star \star \star}$ & 0.16 \\
\hline Yes $(n)$ & 142 & 32 & & \\
\hline \multicolumn{5}{|c|}{ DEPRESSIVE DISORDER } \\
\hline No $(n)$ & 321 & 5 & $27.7^{\star \star \star}$ & 0.20 \\
\hline Yes $(n)$ & 56 & 10 & & \\
\hline \multicolumn{5}{|c|}{ ANY ANXIETY DISORDER } \\
\hline No $(n)$ & 258 & 4 & $29.0^{\star \star \star}$ & 0.17 \\
\hline Yes $(n)$ & 110 & 20 & & \\
\hline
\end{tabular}

DSM, Diagnostic and Statistical Manual of Mental Disorders. ${ }^{\star \star *} p<0.001$.

correctly identified by neurologists (see Table 3). For depression, sensitivity of doctors' diagnoses was $15.2 \%$, specificity was $98.5 \%$. Rates were similar for anxiety disorders, with a sensitivity index of $15.4 \%$ and specificity of $98.5 \%$.

\section{DISCUSSION}

We investigated neurologists' awareness of psychiatric comorbidities in patients with vertigo and dizziness symptoms at their first examination at a tertiary care center specialized in vertigo and balance disorders. Our main finding was that the recognition of psychiatric disorders amongst neurologists in routine clinical practice is very low with only about $10 \%$ of all patients being diagnosed with a psychiatric disorder by the neurologist vs. about $44 \%$ fulfilling DSM-IV criteria. Further, only $15 \%$ of depressive and anxiety disorders were identified correctly, i.e., in accordance with DSM-IV criteria, among all patients. On one hand, corresponding specificity rates were high, indicating that when the neurologists make a psychiatric diagnosis, it mostly is correct. On the other hand, however, doctors in our investigation had a tendency not to give or not to document psychiatric diagnoses in a high number of cases as depicted by the low sensitivity rates.

Our findings of low agreement between SCID-I and doctors' ratings relate to previous studies in neurology $(8,9)$ and other medical fields (10-12) as well as to meta-analytic findings (23). Agreement rates also correspond to findings comparing standardized and unstandardized methods concerning patients with psychiatric disorders (24-28). Standardized interviews such as the SCID-I are seen as the "gold standard" to diagnose mental disorders (29). However, they are highly time as well as cost consuming and thus may not be conducted as a routine diagnostic interview in primary, secondary, or tertiary care. This was also true for the primary routine diagnostics in the current study.

It is important to note that the study was conducted in routine clinical practice, i.e., neurologists were trained to screen for relevant mental disorders, but not explicitly instructed to regularly perform a related questionnaire. Thus, it may be that neurologists were aware of mental psychopathology and simply did not note their impression in the file, especially when a structural dysfunction was found. Moreover, there were structural barriers for the documentation of psychiatric disorders in routine clinical practice. Aspects of psychopathology may have been described in the doctor's report but were not captured by the current study since only actual diagnoses were evaluated. Hence, although our results likely underestimate the diagnostic competency of neurologists during their clinical practice in a tertiary care center, an adequate testing for psychiatric comorbidities and disorders is mandatory and must be included in the diagnostic workflow. In this regard, one has to differentiate between secondary and tertiary care. In secondary care, one would likely assume that a neurologist is practicing within the standards of neurological care even if psychiatric diagnoses are missed in individual patients. In tertiary care, however, the finding that mental health comorbidities that affect $30-50 \%$ of patients are very often not detected is concerning. As it is not realistic to perform detailed standardized interviews for mental disorders by a psychiatrist or psychologist in a routine setting, one solution to this issue could be the implementation of a screening procedure by application of a validated, standardized short questionnaire (e.g., Beck Depression Inventory [BDI; (30)], Beck Anxiety Inventory [BAI; (31)], Hospital Anxiety and Depression Scale [HADS; (32)], Somatic Symptom Disorder-B Criteria Scale [SSD-12; (33)]). This could help to improve the awareness of mental disorders and the rate of early diagnosis and treatment. However, it goes without saying that self-report screening questionnaires do not lead to greater awareness of and better care for mental health comorbidities without equally implementing changes in clinical workflow in order to ensure that mental health symptoms are adequately dealt with. In this regard, research in cardiovascular 
care has shown that screening for depression does not improve outcome for heart failure patients $(34,35)$. Further, a review on recommendations for depression screening in primary care found that patients who undergo screening do not have better outcomes (36) and authors of a meta-analytic study on screening tools for depression in non-mental health settings recommend only using these tools if appropriate organizational strategies and modifications are available (37). Similar findings have been made in oncology, where evidence suggests that a positive screening for psychological distress often does not lead to appropriate care, although a referral is mandatory for the screening to be effective in improving patients' psychological outcome (38). A strategy to better deal with mental health comorbidities in somatic care in general and neurological care specifically could be collaborative care approaches. Clinical guidelines for managing functional symptoms already recommend a collaborative stepped care approach in which the primary care physician coordinates treatment procedures (39). Collaborative care concepts have been implemented and evaluated for depression and somatoform disorders, results indicate that they are superior to treatment as usual $(13,40)$. For patients with somatoform and functional disorders, the evaluation of a pre- and post-intervention in primary care indicated that the investigated interdisciplinary network (The Network for Somatoform and Functional Disorders, Sofu-Net) was feasible and helped to improve doctor-patient communication about psychosocial distress (14). These collaborative care approaches have in common that they have their starting point in primary care. Patients at our center are usually referred from primary to secondary care, the latter then refers to our tertiary treatment. In contrast, an example of a stepped collaborative care concept for our patient group analogous to the Sofu-Net (14) could look as follows. The patient would first visit the primary care physician (PCP). Based on this visit, the PCP could decide to refer to secondary care (e.g., a neurologist). After this, PCP and secondary care physician would discuss the findings and could then decide to (a) observe the symptoms over a defined period of time or (b) refer the patient to our center. Patients could be screened for mental health comorbidities at any of these stages, but at the latest at our center. If the screening is positive, the PCP should be informed and initiate appropriate treatment, i.e., next to somatic care, psychotherapeutic treatment should be initiated. This would require a care network that enables the PCP to refer patients to psychotherapy and be informed about the progress of therapy. This concept could apply to patients presenting with both chronic and acute symptoms and would ensure that patients with chronic complaints are always screened for mental comorbidity. Those with acute symptoms would likely

\section{REFERENCES}

1. Best C, Eckhardt-Henn A, Diener G, Bense S, Breuer P, Dieterich M. Interaction of somatoform and vestibular disorders. J Neurol Neurosurg Psychiatry (2006) 77:658-64. doi: 10.1136/jnnp.2005.072934

2. Eckhardt-Henn A, Tschan R, Best C, Dieterich M. [Somatoform vertigo syndrome]. Nervenarzt (2009) 80:909-17. doi: 10.1007/s00115-009-2736-y more rarely present at the tertiary center. If they do present, they would also be screened for psychopathology which would help to recognize the symptoms at an early stage and therefore simplify access to treatment if necessary, even if the vertigo and dizziness symptoms resolve after a relatively short period of time.

The advantage of the current study is that we examined a large sample size and used multiple sources of information and diagnostic assessment methods such as routine clinical workups and standardized interviews for mental disorders. However, there are some limitations: Our sample is representative for a specialized tertiary care center but not for all patients with vertigo and dizziness. Thus, a selection bias must be assumed with respect to chronification and a higher rate of psychiatric disorders.

In conclusion, our results show that neurologists' awareness of psychiatric disorders in routine clinical practice in a specialized tertiary care center for vertigo and dizziness is low. This is in accordance with previous studies in both similar as well as different medical fields. However, particularly since psychiatric disorders tend to take a chronic course if not recognized early $(6,7)$, diagnosis of psychopathology should be given more importance and appropriate concepts of care, such as stepped collaborative care, should be implemented.

\section{DATA AVAILABILITY STATEMENT}

The raw data supporting the conclusions of this manuscript will be made available by the authors, without undue reservation, to any qualified researcher.

\section{AUTHOR CONTRIBUTIONS}

The study was designed by GS-M, CL, MD, PH, and SB-B. KL, GS-M, SB-B, and KR led the data collection. KL conducted the statistical analysis and wrote all drafts. AD, HS, GS-M, and KR contributed to the analysis strategy and all drafts. All authors contributed to the interpretation of the results. All authors critically reviewed the manuscript and approved the final version. All authors agree to be accountable for all aspects of the work in ensuring that questions related to the accuracy or integrity of any part of the work are appropriately investigated and resolved.

\section{FUNDING}

Parts of this project were supported by funds from the German Federal Ministry of Education and Research under Grant code 01 EO 0901. 
5. Dieterich M, Staab JP. Functional dizziness: from phobic postural vertigo and chronic subjective dizziness to persistent postural-perceptual dizziness. Curr Opin Neurol. (2017) 30:107-13. doi: 10.1097/WCO.0000000000000417

6. Radziej K, Schmid-Mühlbauer G, Limburg K, Lahmann C. [Tailored care for functional vertigo/dizziness - an integrative group psychotherapy approach]. Psychother Psychosom Med Psychol. (2017) 67:245-51. doi: 10.1055/s-0043-104768

7. Wittchen H-U, Mühlig S, Beesdo K. Mental disorders in primary care. Dialogues Clin Neurosci. (2003) 5:115.

8. Hesdorffer DC. Comorbidity between neurological illness and psychiatric disorders. CNS Spectr. (2016) 21:230-8. doi: 10.1017/S1092852915000929

9. Bouwmans AE, Weber WE. Neurologists' diagnostic accuracy of depression and cognitive problems in patients with parkinsonism. BMC Neurol. (2012) 12:37. doi: 10.1186/1471-2377-12-37

10. Söllner W, DeVries A, Steixner E, Lukas P, Sprinzl G, Rumpold G, et al. How successful are oncologists in identifying patient distress, perceived social support, and need for psychosocial counselling? Br J Cancer (2001) 84:179-85. doi: 10.1054/bjoc.2000.1545

11. Mitchell AJ, Meader N, Bird V, Rizzo M. Clinical recognition and recording of alcohol disorders by clinicians in primary and secondary care: meta-analysis. Br J Psychiatry J Mental Sci. (2012) 201:93-100. doi: 10.1192/bjp.bp.110.091199

12. Topitz A, Benda N, Saumer G, Friedrich F, König D, Soulier N, et al. Prevalence and recognition of depression among inpatients of non-psychiatric hospital departments. Neuropsychiatry (2015) 29:63-70. doi: 10.1007/s40211-015-0145-y

13. van der Feltz-Cornelis CM, Van Os TW, Van Marwijk HW, Leentjens AF. Effect of psychiatric consultation models in primary care. A systematic review and meta-analysis of randomized clinical trials. J Psychosom Res. (2010) 68:521-33. doi: 10.1016/j.jpsychores.2009.10.012

14. Shedden-Mora M, Gross B, Lau K, Gumz A, Wegscheider K, Löwe B. Collaborative stepped care for somatoform disorders: a pre-postintervention study in primary care. J Psychosom Res. (2016) 80:23-30. doi: 10.1016/j.jpsychores.2015.11.004

15. Wittchen H, Wunderlich U, Gruschwitz S, Zaudig M. SCID I: Structured Clinical Interview for DSM-IV: Axis I: Mental Disorders. Göttingen: Hogrefe (1997).

16. Schmid G, Henningsen P, Dieterich M, Sattel H, Lahmann C. Psychotherapy in dizziness: a systematic review. J Neurol Neurosurg Psychiatry. (2011) 82:601-6. doi: 10.1136/jnnp.2010.237388

17. Limburg K, Sattel H, Radziej K, Lahmann C. DSM-5 somatic symptom disorder in patients with vertigo and dizziness symptoms. J Psychosom Res. (2016) 91:26-32. doi: 10.1016/j.jpsychores.2016.10.005

18. Lahmann C, Henningsen P, Dieterich M, Feuerecker R, Cyran CA, Schmid G. The munich diagnostic and predictor study of dizziness: objectives, design, and methods. J Neurol. (2012) 259:702-11. doi: 10.1007/s00415-011-6250-3

19. World Health Organization. Internationale Klassifikation psychischer Störungen ICD-10, Kapitel V (F) Klinisch-diagnostische Leitlinien. In: Dilling H, Mombour W, and Schmidt MH, editors. Bern: Huber (2013).

20. American Psychiatric Association. Diagnostic and Statistical Manual of Mental Disorders. 5th edn. Washington, DC: American Psychiatric Association (2013).

21. Cohen J. A coefficient of agreement for nominal scales. Educ Psychol Meas. (1960) 20:37-46. doi: 10.1177/001316446002000104

22. Lenhard W, Lenhard A. Berechnung von Testgütekriterien für Screeningverfahren. Bibergau: Psychometrica (2014). Available online at: http://www.psychometrica.de/testkennwerte.html

23. Cepoiu M, McCusker J, Cole MG, Sewitch M, Belzile E, Ciampi A. Recognition of depression by non-psychiatric physicians-a systematic literature review and meta-analysis. J Gen Intern Med. (2008) 23:25-36. doi: 10.1007/s11606-007-0428-5

24. Egan S, Nathan P, Lumley M. Diagnostic concordance of ICD-10 personality and comorbid disorders: a comparison of standard clinical assessment and structured interviews in a clinical setting. Aust N Z J Psychiatry (2003) 37:484-91. doi: 10.1046/j.1440-1614.2003.01226.x
25. Kashner TM, Rush AJ, Surís A, Biggs MM, Gajewski VL, Hooker DJ, et al. Impact of structured clinical interviews on physicians' practices in community mental health settings. Psychiatr Serv. (2003) 54:712-8. doi: 10.1176/appi.ps.54.5.712

26. Miller PR. Inpatient diagnostic assessments: 2. Interrater reliability and outcomes of structured vs. unstructured interviews. Psychiatry Res. (2001) 105:265-71. doi: 10.1016/S0165-1781(01)00318-3

27. Miller PR, Dasher R, Collins R, Griffiths P, Brown F. Inpatient diagnostic assessments: 1. Accuracy of structured vs. unstructured interviews. Psychiatry Res. (2001) 105:255-64. doi: 10.1016/S0165-1781(01)00317-1

28. Shear MK, Greeno C, Kang J, Ludewig D, Frank E, Swartz HA, et al. Diagnosis of non-psychotic patients in community clinics. Am J Psychiatry (2000) 157:581-7. doi: 10.1176/appi.ajp.157.4.581

29. Andreas S, Theisen P, Mestel R, Koch U, Schulz H. Validity of routine clinical DSM-IV diagnoses (Axis I/II) in inpatients with mental disorders. Psychiatry Res. (2009) 170:252-5. doi: 10.1016/j.psychres.2008.09.009

30. Beck AT, Steer RA, Brown GK. Beck Depression Inventory-II. San Antonio, TX: Psychological Corporation (1996).

31. Beck AT, Steer RA. Manual for the Beck Anxiety Inventory. San Antonio, TX: Psychological Corporation (1990).

32. Snaith RP. The hospital anxiety and depression scale. Health Quality Life Outcomes (2003) 1:29. doi: 10.1186/1477-7525-1-29

33. Toussaint A, Murray AM, Voigt K, Herzog A, Gierk B, Kroenke $\mathrm{K}$, et al. Development and validation of the somatic symptom disorder-B criteria scale (SSD-12). Psychosom Med. (2016) 78:5-12. doi: 10.1097/PSY.0000000000000240

34. Thombs BD, De Jonge P, Coyne JC, Whooley MA, Frasure-Smith N, Mitchell $\mathrm{AJ}$, et al. Depression screening and patient outcomes in cardiovascular care: a systematic review. JAMA (2008) 300:2161-71. doi: 10.1001/jama. 2008.667

35. Thombs BD, Roseman M, Coyne JC, de Jonge P, Delisle VC, Arthurs E, et al. Does evidence support the American Heart Association's recommendation to screen patients for depression in cardiovascular care? An updated systematic review. PLoS ONE (2013) 8:e52654. doi: 10.1371/journal.pone. 0052654

36. Thombs BD, Coyne JC, Cuijpers P, De Jonge P, Gilbody S, Ioannidis JP, et al. Rethinking recommendations for screening for depression in primary care. CMAJ (2012) 184:413-8. doi: 10.1503/cmaj.111035

37. Gilbody S, Sheldon T, House A. Screening and case-finding instruments for depression: a meta-analysis. CMAJ (2008) 178:997-1003. doi: $10.1503 / \mathrm{cmaj} .070281$

38. Mitchell AJ. Screening for cancer-related distress: when is implementation successful and when is it unsuccessful? Acta Oncol. (2013) 52:216-24. doi: 10.3109/0284186X.2012.745949

39. Schaefert R, Hausteiner-Wiehle C, Häuser W, Ronel J, Herrmann M, Henningsen P. Clinical practice guideline: non-specific, functional and somatoform bodily complaints. Dtsch Arztebl Int. (2012) 109:803-13. doi: 10.3238/arztebl.2012.0803

40. Sighinolfi C, Nespeca C, Menchetti M, Levantesi P, Murri MB, Berardi D. Collaborative care for depression in European countries: a systematic review and meta-analysis. J Psychosom Res. (2014) 77:247-63. doi: 10.1016/j.jpsychores.2014.08.006

Conflict of Interest Statement: The authors declare that the research was conducted in the absence of any commercial or financial relationships that could be construed as a potential conflict of interest.

Copyright (c) 2018 Limburg, Dinkel, Schmid-Mühlbauer, Sattel, Radziej, BeckerBense, Henningsen, Dieterich and Lahmann. This is an open-access article distributed under the terms of the Creative Commons Attribution License (CC BY). The use, distribution or reproduction in other forums is permitted, provided the original author(s) and the copyright owner(s) are credited and that the original publication in this journal is cited, in accordance with accepted academic practice. No use, distribution or reproduction is permitted which does not comply with these terms. 